\title{
Singularity Analysis of a Variant of the Painlevé-Ince Equation
}

\author{
Amlan K Halder* \\ Department of Mathematics, Pondicherry \\ University, Kalapet, India-605014 \\ Andronikos Paliathanasis ${ }^{\dagger}$ \\ Institute of Systems Science, Durban University of Technology \\ Durban 4000, Republic of South Africa. \\ PGL Leach ${ }^{\ddagger}$ \\ School of Mathematics, Statistics and Computer Science, \\ University of KwaZulu-Natal, Durban, South Africa. \\ Institute of Systems Science, Durban University of Technology \\ Durban 4000, Republic of South Africa.
}

June 4, 2019

\begin{abstract}
We examine by singularity analysis an equation derived by reduction using Lie point symmetries from the Euler-Bernoulli Beam equation which is the Painlevé-Ince Equation with additional terms. The equation possesses the same leading-order behaviour and resonances as the Painlevé-Ince Equation and has a Right Painlevé Series. However, it has no Left Painlevé Series. A conjecture for the existence of Left Painlevé Series for ordinary differential equations is given.

Keywords: Singularity analysis; Painlevé-Ince; Integrability
\end{abstract}

\section{Introduction}

The Painlevé-Ince Equation

$$
y^{\prime \prime}+3 y y^{\prime}+y^{3}=0,
$$

where the prime denotes differentiation of the dependent variable, $y(x)$, with respect to the independent variable, $x$, is an equation noted for its interesting properties. Firstly it possesses eight Lie point symmetries [14] with the Lie Algebra $\operatorname{sl}(2, R)$ which means that it is linearisable by a point transformation. Secondly it is the second member of the Riccati Hierarchy which is based upon the Riccati Equation with the recursion operator $D+y$, where $D$ is the operator $\frac{d}{d y}[\underline{8}$. Thirdly it possesses the Painlevé Property in a very unique way in that there are two possible Laurent expansions about its simple pole. The coefficient of the leading-order term can be 1 or 2 . For the former the resonances are the generic -1 and 1 . For the latter the resonances are again -1 and an unexpected -2 . This meant that the Laurent expansion must be decreasing in exponent from the simple pole [13. The existence of a resonance at an exponent lower than the exponent of the leading-order term was not expected and the proposal was derided by some, but the careful analysis of Feix and his team [9, 10, 11] established the sense with the addition of the Left Painlevé Series to the well-established Right

\footnotetext{
*Email: amlan.haldar@yahoo.com

${ }^{\dagger}$ Email: anpaliat@phys.uoa.gr

‡Email: leachp@ukzn.ac.za
} 
Painlevé Series. One notes that Andriopoulos et al [4] discussed the existence of both positive and negative nongeneric resonances for higher-order equations, ie Mixed Painlevé Series and their geometric interpretation.

In an analysis of the Euler-Bernoulli Equation for a beam Halder et al 12 determined the Lie point symmetries 1 and used them initially to reduce the fourth-order partial differential equation to a fourth-order ordinary differential equation. This fourth-order equation had sufficient Lie point symmetries to reduce it to a second-order equation devoid of Lie point symmetries. It was noted that the second-order equation was the Painlevé-Ince Equation with some rather messy additional terms of order lower than the second order in the derivative. It is the purpose of this paper to demonstrate that this equation does in fact satisfy the requirement of the ARS algorithm [1, 2, 3]. The equation is nonautonomous and so we treat it in the spirit of the treatment of nonautonomous equations as found in Andriopoulos et al [5].

\section{The equation and its singularity analysis}

The nonlinear second-order ordinary differential equation of our consideration is

$$
y^{\prime \prime}+y^{\prime}\left(\frac{7}{x}+3 y\right)+y^{3}+\frac{7 y^{2}}{x}+y\left(\frac{1}{16 a b x^{4}}+\frac{39}{4 x^{2}}\right)+\frac{3}{2 x^{3}}=0,
$$

where the symbolic usages are the same as for (11) above. As, mentioned in the introduction, equation (2) caught our attention, as it is obtained from the reduction of the well-known Euler-Bernoulli Beam equation, which is something new according to the author's knowledge and cannot be found in the literature. A first glance at the equation establish the fact that, it is a variant of the Painlevé-Ince equation. It has zero Lie-point symmetries, and we choose to study the singularity analysis to ascertain its integrability. A problem of interests also in the area of physics where the Euler-Bernoulli Beam equation has applications. The similarities and dissimilarities of equation (2) with the Painlevé-Ince equation have been mentioned subsequently.

To determine the leading-order behaviour of equation (2), we substitute

$$
y=a\left(x-x_{0}\right)^{p},
$$

where $x_{0}$ is the location of the putative singularity and, being movable, is one of the constants of integration to be determined from the initial conditions, into (2) to obtain

$$
\begin{aligned}
0= & \frac{3 x^{4}}{2}-a p x^{7}\left(x-x_{0}\right)^{-2+p}+a p^{2} x^{7}\left(x-x_{0}\right)^{-2+p}+7 a p x^{6}\left(x-x_{0}\right)^{-1+p} \\
& +\frac{x^{3}\left(x-x_{0}\right)^{p}}{16 b}+\frac{39}{4} a x^{5}\left(x-x_{0}\right)^{p}+7 a^{2} x^{6}\left(x-x_{0}\right)^{2 p}+ \\
& +a^{3} x^{7}\left(x-x_{0}\right)^{3 p}+3 a^{2} p x^{7}\left(x-x_{0}\right)^{-1+2 p} .
\end{aligned}
$$

We consider the dominant terms to compute the value of $p$, which is,

$$
-a p x^{7}\left(x-x_{0}\right)^{-2+p}+a p^{2} x^{7}\left(x-x_{0}\right)^{-2+p}+a^{3} x^{7}\left(x-x_{0}\right)^{3 p}+3 a^{2} p x^{7}\left(x-x_{0}\right)^{-1+2 p},
$$

we equate the exponents of the dominant terms, which are $-2+p, 3 p$ and $-1+2 p$, from which it is evident that $p=-1$, ie, the singularity is a simple pole.

We solve the dominant terms

$$
\frac{2 a x^{7}}{\left(x-x_{0}\right)^{3}}-\frac{3 a^{2} x^{7}}{\left(x-x_{0}\right)^{3}}+\frac{a^{3} x^{7}}{\left(x-x_{0}\right)^{3}}=0,
$$

for $a$ and obtain $a=0,1,2$. So far all is the same as for (11). To determine the resonances for the leading-order coefficient $a=1$ we make the substitution

$$
y=\left(x-x_{0}\right)^{-1}+m\left(x-x_{0}\right)^{-1+s},
$$

into (2), take the coefficient of $m$ and require it to vanish for the terms corresponding to the dominant terms from which $p$ was found.

\footnotetext{
${ }^{1}$ The Mathemaica add-on Sym [6, 7] was used to obtain the symmetries.
} 
The values of $s$ so found are -1 and 1 . The former value of $s$, which is -1 , is generic in nature and is related to the arbitrariness of the location of the movable singularity. The details regarding the various values of the resonances and most importantly the implications of the value -1 is explained in [5].

When we use the value $a=2$, the resonances are -1 and -2 . Thus far the results are as for the Painlevé-Ince Equation, (11).

To determine consistency we need to substitute a (truncated) Laurent expansion into the full equation, which is,

$$
\frac{1}{\left(x-x_{0}\right)}+a_{0}+a_{1}\left(x-x_{0}\right)+a_{2}\left(x-x_{0}\right)^{2}+a_{3}\left(x-x_{0}\right)^{3},
$$

where, $a_{0}, a_{1}, a_{2}$ and $a_{3}$ are the coefficients of the series terms. For the coefficient constants $a_{0}, a_{1}, a_{2}$ and $a_{3}$ in the Laurent expansion we obtain the following results

$$
\begin{aligned}
& a_{1}=\frac{-1-156 b x_{0}^{2}-224 a_{0} b x_{0}^{3}-48 a_{0}^{2} b x_{0}^{4}}{48 b x_{0}^{4}}, \\
& a_{2}=\frac{11+2 a_{0} x_{0}+1380 b x_{0}^{2}+2104 a_{0} b x_{0}^{3}+896 a_{0}^{2} b x_{0}^{4}+128 a_{0}^{3} b x_{0}^{5}}{128 b x_{0}^{5}} \text { and } \\
& a_{3}=\frac{-\left(1+8 b x_{0}^{2}\left(372+173 a_{0} x_{0}+30 a_{0}^{2} x_{0}^{2}\right)+80 b^{2} x_{0}^{4}(4095+\ldots)\right)}{11520 b^{2} x_{0}^{8}} .
\end{aligned}
$$

One could continue further, but the successive coefficients in the Right Painlevé Series are expressible in terms of the two arbitrary constants, $x_{0}$ and $a_{0}$, which is as they should be.

We turn now to the second possibility, $a=2$, for the value of the coefficient of the leading-order term for which the resonances are -1 and -2 . We substitute

$$
y=2\left(x-x_{0}\right)^{-1}+a_{2}\left(x-x_{0}\right)^{-2}+a_{3}\left(x-x_{0}\right)^{-3}+a_{4}\left(x-x_{0}\right)^{-4}+a_{5}\left(x-x_{0}\right)^{-5},
$$

in equation (2), to check the consistency by computing the coefficients of the Laurent expansion.

An attempt to put various coefficients to zero fails immediately due to the presence of the term, $x^{4}$, in (2) for which there is no compensating term in the posited Left Painlevé Series.

\section{Conclusion}

The Painlevé-Ince Equation is the first known instance of an ordinary differential equation possessing both Left and Right Painlevé Series. Equation (2), obtained by reduction using Lie point symmetries from the Euler-Bernoulli Beam equation, is like a Painlevé-Ince Equation with some additional terms. These additional terms do not affect the first two steps of the ARS algorithm as they are not part of the dominant terms in (2). However, when it comes to the test for consistency, these additional terms must play a role.

The basis of the singularity analysis is the existence of a Laurent expansion about a singularity. There are three possible forms for a convergent Laurent expansion. One is an expansion from singularity in increasing powers of $\left(x-x_{0}\right)$, where $x_{0}$ is the location of the singularity in the complex plane. This is the well-known rightPainlevé series. Secondly the series can descend in powers from the singularity and one has the left-Painlev'e series. Thirdly there can be an expansion between two singularities which gives rise to the mixed-Painlevé series. All of these are well illustrated in [15. One should bear in mind that the existence of, say, a left-Painlevé series does suggest that the series, of the nature of an asymptotic series, is convergent over the rest of the complex plane. On the other hand the mixed series gives a starting point for a series which cannot be convergent over the whole complex plane and so it must terminate at the boundary determined by another singularity.

As far as the equation of our problem is concerned, for the Right Painlevé Series there is no serious effect apart from making the coefficients of the terms in the series more complex. Nevertheless those coefficients are expressible in terms of the two constants of integration required for the second-order equation. We note that the location of the movable pole plays an active role in the further coefficients as predicted in [5].

The collapse of the Left Painlevé Series comes as no surprise in retrospect as the negative powers cannot possibly balance positive powers. Indeed it suggests strongly that an equation with nondominant terms might not be able to possess a Left Painlevé Series.

Conjecture: For a differential equation to possess a Left Painlevé Series all terms must be dominant. 


\section{Acknowledgements}

AKH expresses grateful thanks to UGC (India) NFSC, Award No. F1-17.1/201718/RGNF-2017-18-SC-ORI39488 for financial support and Late Prof. K.M.Tamizhmani for the discussions which AKH had with him which formed the basis of this work. PGLL acknowledges the support of the National Research Foundation of South Africa, the University of KwaZulu-Natal and the Durban University of Technology and thanks the Department of Mathematics, Pondicherry University, for gracious hospitality.

\section{References}

[1] Ablowitz MJ, Ramani A \& Segur H (1978) Nonlinear Evolution Equations and Ordinary Differential Equations of Painlevé Type Lett Nuovo Cimento 23 333-337.

[2] Ablowitz MJ, Ramani A \& Segur H (1980) A connection between nonlinear evolution equations and ordinary differential equations of P-type I Journal of Mathematical Physics 21 715-721.

[3] Ablowitz MJ, Ramani A \& Segur H (1980) A connection between nonlinear evolution equations and ordinary differential equations of P-type II Journal of Mathematical Physics 21 1006-1015.

[4] Andriopoulos K \& Leach PGL (2006) An interpretation of the presence of both positive and negative nongeneric resonances in the singularity analysis Physics Letters A 359 199-203.

[5] Andriopoulos K \& Leach PGL (2011) Singularity analysis for autonomous and nonautonomous differential equations Applicable Analysis and Discrete Mathematics 5 230-239.

[6] Dimas S \& Tsoubelis D (2004, October) SYM: A new symmetry-finding package for Mathematica In Proceedings of the 10th International Conference in Modern Group Analysis (University of Cyprus Press) 64-70.

[7] Dimas S \& Tsoubelis D (2006, June) A new Mathematica-based program for solving overdetermined systems of PDEs In 8th International Mathematica Symposium.

[8] Euler M, Euler N \& Leach PGL (2007) The Riccati and Ermakov-Pinney hierarchies Journal of Nonlinear Mathematical Physics 14 290-310.

[9] Feix MR, Géronimi C, Cairó L, Leach PGL, Lemmer RL \& Bouquet SÉ (1997) On the singularity analysis of ordinary differential equations invariant under time translation and rescaling Journal of Physics A: Mathematical and General 30 7437-7461.

[10] Feix MR, Géronimi C \& Leach PGL (2005) Properties of some autonomous equations invariant under homogeneity symmetries Problems of Nonlinear Analysis in Engineering Systems 11 26-34.

[11] Géronimi C, Leach PGL \& Feix MR (2002) Singularity analysis and a function unifying the Painlevé and $\Psi$ series Journal of Nonlinear Mathematical Physics 9 Second Supplement 36-48.

[12] Halder AK, Paliathansis K \& Leach PGL (2019) A Complete Study Of Different Forms Of Beam Equations Through Lie Group Analysissubmitted.

[13] Lemmer RL \& Leach PGL (1993) The Painlevé test, hidden symmetries and the equation $y^{\prime \prime}+y y^{\prime}+k y^{3}=0$ Journal of Physics A: Mathematical and General 26 5017-5024.

[14] Mahomed FM \& Leach PGL (1985) The linear symmetries of a nonlinear differential equation Quaestiones Mathematicae 8 241-274.

[15] Paliathanasis A \& Leach PGL (2016) Nonlinear Ordinary Differential Equations: A discussion on Symmetries and Singularities International Journal Geometric Methods Modern Physics 131630009 